\title{
A three-dimensional robust seismic ray tracer for volcanic regions
}

\author{
Kiyoshi Nishi \\ Sakurajima Volcano Research Center, DPRI, Kyoto University, Sakurajima-cho, Kagoshima 891-1419, Japan
}

(Received March 9, 2000; Revised December 12, 2000; Accepted December 21, 2000)

\begin{abstract}
Seismic velocity structure of volcanic region is highly heterogeneous so that seismic ray tracer used in this field should be robust for the velocity heterogeneity. From this view point a three-dimensional robust seismic ray tracer, effective in any complicated velocity structure, is developed by using a hybrid scheme of the shortest path calculation and the downhill simplex optimization method. The node configuration necessary for the three-dimensional shortest path calculation is newly presented. Validity and efficiency of the calculation are examined by synthetic tests. A travel time accuracy of less than $0.1 \%$, and a rms ray path error of $0.05 \mathrm{~km}$ are achieved. Calculations for the synthetic velocity models and checkerboard testing show the effectiveness of this ray tracer in practical situations. The present ray tracer is recommended to be used in travel time tomography in highly heterogeneous velocity structure such as volcanic regions.
\end{abstract}

\section{Introduction}

The seismic ray tracer is a tool for obtaining ray paths and travel times of seismic waves in a given velocity structure. It is essential in forward modeling, such as hypocenter determination and travel time tomography. The seismic velocity structure in volcanic regions is highly heterogeneous. The seismic ray tracer used in volcanic areas should consequently be robust to this heterogeneity. The present paper provides a robust three-dimensional seismic ray tracer applicable to observation in volcanic regions.

There are four kinds of algorithms used in existing ray tracers. These are shooting, bending, finite-difference and the graph theory (the shortest path calculation). Of these, the finite-difference method and the method by the graph theory are considered to be robust to heterogeneous velocity structure.

The finite-difference method solves the eikonal equation by using numerical schemes such as the Runge-Kutta method and wave front expansion (Reshef and Kosloff, 1986; Vidale, 1988; van Trier and Symes, 1991; Podvin and Lecomte, 1991). When accuracy and stability are required, this method becomes similar to the method by the graph theory based on Fermat's principle (Klimeš and Kvasnička, 1994). It also loses simplicity and becomes expensive (Zhang and Toksöz, 1998). Comparisons of the finite-difference method and graph theory are found in Klimeš and Kvasnička (1994) and Zhang and Toksöz (1998); they suggest the superiority of ray tracing by the graph theory.

Nakanishi and Yamaguchi (1986) applied the idea of the shortest path (in the graph theory) to seismic ray tracing. This method regards the solution for the shortest path over a network as satisfying the Fermat's principle of stationary time for wave propagation. The basic principle is straightforward

Copy right (C) The Society of Geomagnetism and Earth, Planetary and Space Sciences (SGEPSS); The Seismological Society of Japan; The Volcanological Society of Japan; The Geodetic Society of Japan; The Japanese Society for Planetary Sciences. and guarantees a stable global minimum travel time while requiring no initial value and no derivative calculation. A concise explanation on fundamental concept of this method, and the calculation scheme for the first arrival and the latter phases, are found in Moser (1991).

Moser (1991) evaluated the errors in this method and demonstrated its superiority. He also recommended using the heap data structure, following Gallo and Pallottino (1986) in the shortest path calculation via Dijkstra's algorithm (Dijkstra, 1959). Since the work of Nakanishi and Yamaguchi (1986), several improvements have increased the accuracy and the efficiency of the calculation (e.g., Saito, 1989, 1990; Moser, 1991; Fisher and Lees, 1993; Klimeš and Kvasnička, 1994; Cheng and House, 1996; Zhang and Toksöz, 1998).

Reliability of the tomographic results is sensitive to numerical noise introduced in the forward calculation (Fischer and Lees, 1993). Furthermore, calculations of more than thousands of seismic rays are involved. Robustness under velocity heterogeneity is guaranteed from Dijkstra's algorithm for the shortest path calculation. However, the efficiency of the calculation using existing shortest path ray tracers is insufficient for practical use in applications such as travel time tomography.

\section{Methods}

The error in the ray tracer using the shortest path calculation depends on the number of rays connected to the node (angle resolution) and the size of the block (space resolution); these two errors are independent (Moser, 1991; Fisher and Lees, 1993). To obtain accurate ray path and travel times, it is therefore important to use the node configuration of multi-connections and a small block size. However, use of small blocks involves an increase in the number of nodes and consequently reduced the efficiency of the calculations. Below, a formulation of the node configuration is presented for 
multi-connections in three-dimensions and a hybrid scheme is proposed for the rapid calculation of the shortest path.

\subsection{Node configuration}

Saito (1990) and Cheng and House (1996) present a threedimensional scheme of the shortest path calculation for ray tracing. In this scheme the nodes are placed only at the vertexes of the blocks. Therefore, high resolution of the ray direction requires connection with the vertex of distant blocks. To construct the ray path by connecting the nodes with faraway blocks is inefficient because of the increase in the number of nodes. Abrupt changes in the velocity structure are also problematic because they require long ray paths of constant velocity.

Alternative schemes of node configuration have been proposed for the two-dimensional problem (e.g., Nakanishi and Yamaguchi, 1986; Moser, 1991; Fisher and Lees, 1993). In this node configuration, nodes are placed on the boundary of each cell, and the ray passes through to the neighbor cell via a node common to each cell. This node configuration requires neither a long ray path nor increases in the number of nodes to give a high-resolution ray path. The corresponding node configuration is formulated for the 3-D problem by the following equations (1). An example of ray paths are shown in Fig. 1.

$$
\begin{aligned}
& \left|x_{v}-x_{i}\right| \leq L \\
& \left|y_{v}-y_{i}\right| \leq L \\
& \left|z_{v}-z_{i}\right| \leq L \\
& \left|x_{v}-x_{j}\right| \leq L \\
& \left|y_{v}-y_{j}\right| \leq L \\
& \left|z_{v}-z_{j}\right| \leq L \\
& \left(x_{v}-x_{i}\right)\left(x_{v}-x_{j}\right) \geq 0 \\
& \left(y_{v}-y_{i}\right)\left(y_{v}-y_{j}\right) \geq 0 \\
& \left(z_{v}-z_{i}\right)\left(z_{v}-z_{j}\right) \geq 0
\end{aligned}
$$

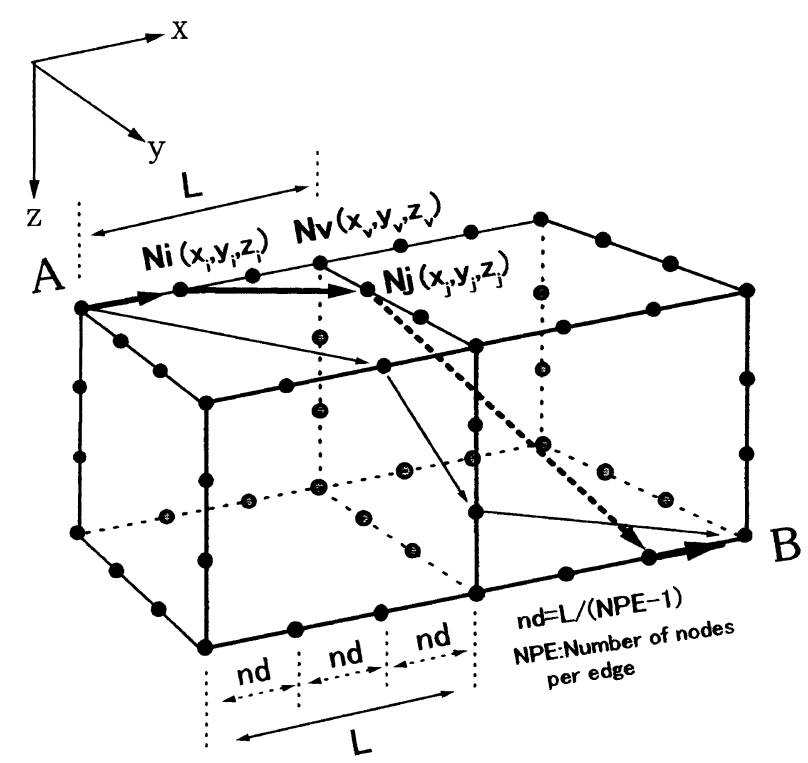

Fig. 1. Node configuration and examples of path (A to B). nd: node distance, NPE: number of nodes per edge. When the travel time of the path indicated by thick arrows is less than that indicated by thin arrows, the former is adopted as the ray path. where

$L:$ the edge length of the block (Fig. 1)

$\left(X_{i}, Y_{i}, Z_{i}\right)$ : coordinate of the node $N_{i}$ (Fig. 1)

$\left(X_{j}, Y_{j}, Z_{j}\right)$ : coordinate of the node $N_{j}$ (Fig. 1) connected to the node $N_{i}$

$\left(X_{v}, Y_{v}, Z_{v}\right)$ : coordinate of a common vertex $N_{v}$ of the blocks to which the nodes $N_{i}$ and $N_{j}$ belong (Fig. 1).

Equations (1) constrain the relation of the nodes that represent the ray path. It is not necessary to make the node distances identical on the edge. In the actual calculation, however, identical distances are adopted for convenience of the code (Fig. 1). The number of nodes per edge (NPE) (Fischer and Lees, 1993) is determined from the accuracy required.

The travel time between two nodes is calculated using the trapezoidal rule, and the velocity of the node is obtained using linear interpolation from surrounding eight vertexes whose velocities are given as part of velocity structure (e.g., Um and Thurber, 1987). Since the travel time between two nodes is defined, we can find the shortest path and its travel time using Dijkstra's algorithm (Moser, 1991).

\subsection{Hybrid scheme with optimization method}

To increase the accuracy of the locus of the ray path and the travel time, the cubic block must be small. This increases the number of nodes and causes inefficiency. To avoid this problem I propose the following hybrid scheme;

Step 1: Find a ray path using Dijkstra's algorithm with the appropriate block size and node configuration.

Step 2: Minimize the travel time of the ray path obtained in Step 1 using conventional optimization methods.

There are many optimization methods including grid-search, gradient-search, the Marquardt method, Monte Carlo techniques, genetic algorithms, neural networks, fuzzy logic. For speed and robustness the simplex method (Nelder and Mead, 1965; Press et al., 1992) will be used here. This method seeks the minimum value of a multi-dimensional function and requires no calculation of derivatives. Calculation time increases with the number of dimensions. However, in threedimensions, this is not severe and is compensated by the stability.

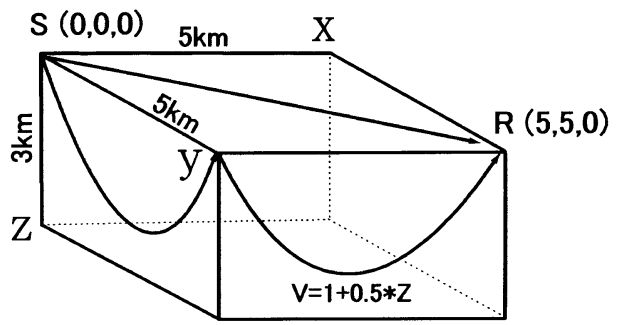

Fig. 2. Velocity structure for the test and a schematic ray path. The velocity depends on depth as $V(z)=1+0.5 * z$, where $V(z)$ is velocity $(\mathrm{km} / \mathrm{s})$ and $z$ is depth $(\mathrm{km})$. 
(a)

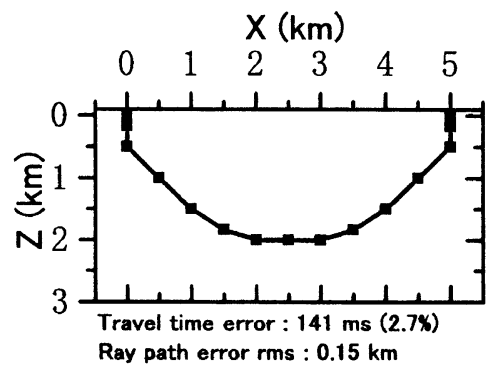

(b)

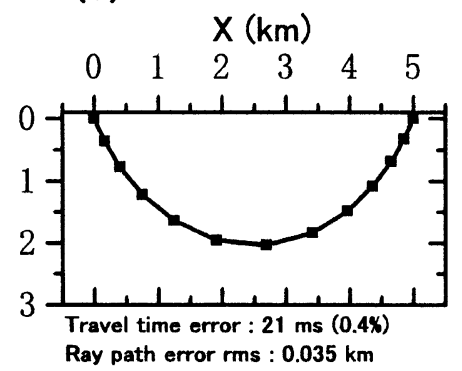

(c)

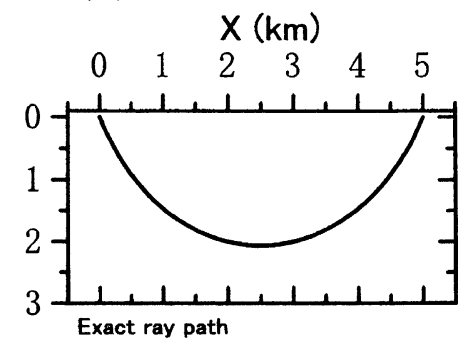

Fig. 3. Results for the velocity structure shown in Fig. 2. (a) Results obtained by shortest path calculation with block size $500 \mathrm{~m}$ and four nodes per edge (NPE). (b) Results of optimization of the ray path in (a) with the simplex method. (c) Ray path obtained by exact solution (e.g., Sheriff and Geldart, 1995).

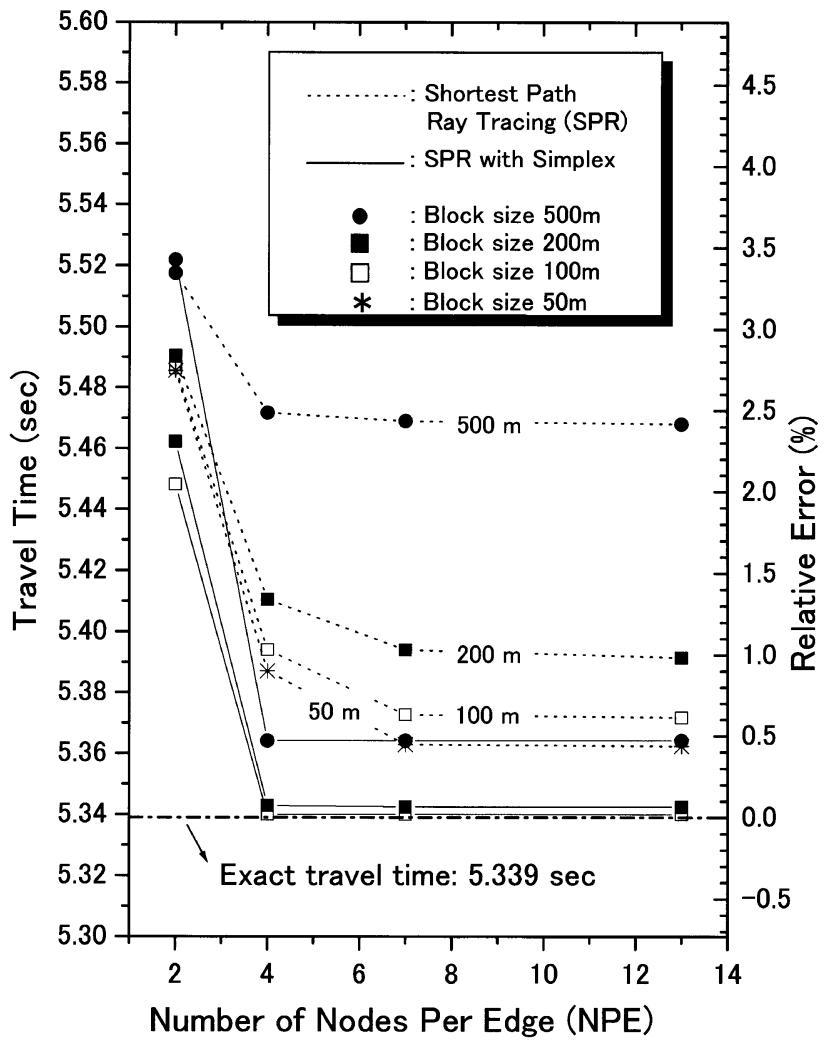

Fig. 4. The travel time and relative error as functions of the number of nodes per edge (NPE) and the block size.

The detailed procedure for the shortest ray path calculation with simplex optimization is as follows;

Step 1: Find the shortest ray path using Dijkstra's algorithm

1-1: Set the node configuration (Block size, NPE)

1-2: Find the nearest neighbor node to the source and the station

1-3: Find the shortest ray path using Dijkstra's algorithm

Step 2: Optimize the travel time using the simplex method

2-1: Construct the node series $N_{1}$ to $N_{n}$, adding the points of the source and the station to the

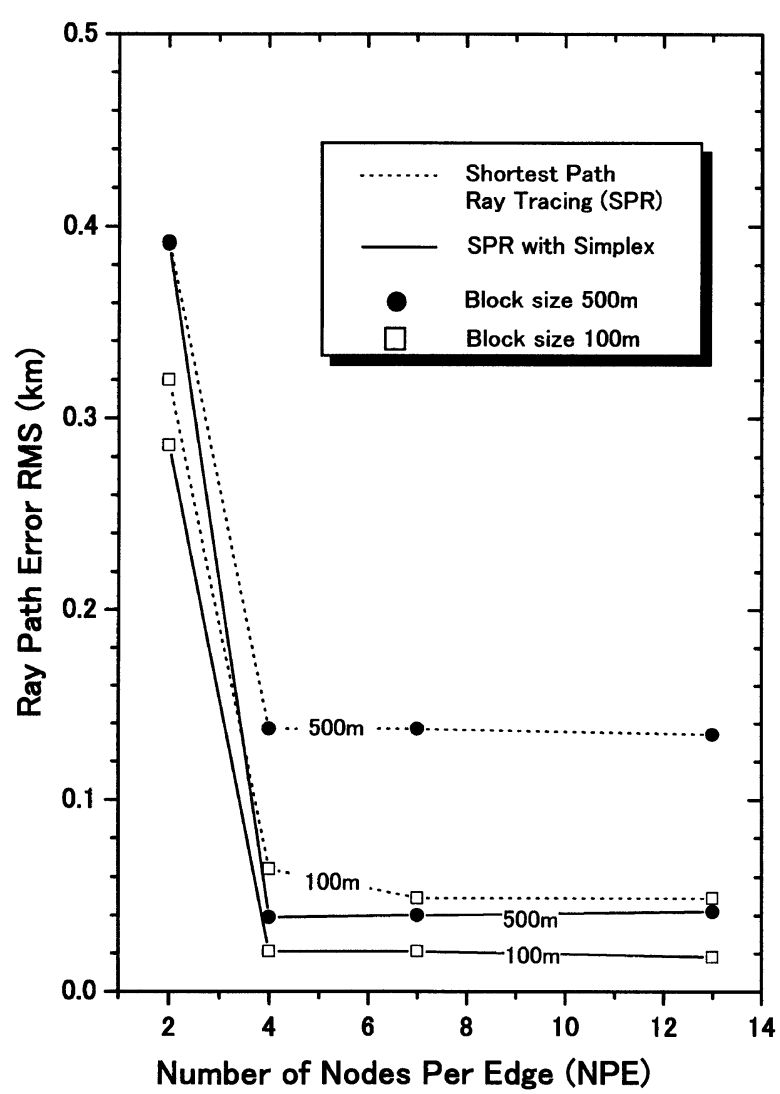

Fig. 5. The rms ray path error as a function of the number of nodes per edge (NPE) and the block size.

nodes that represent the shortest ray path obtained by Step 1-3, where $N_{1}, N_{n}$ represent the source and station respectively

2-2: Calculate the "old" travel time from $N_{1}$ to $N_{n}$

2-3: Set the termination condition of the simplex method (minimum simplex size, maximum number of function evaluations)

2-4: Search the coordinate of the node $N_{i}$ using the simplex method that minimizes the travel time from $N_{i}-1$ to $N_{i}+1$ via $N_{i}$, where $N_{i}-1$ and $N_{i}+i$ are fixed temporally, and then move " $i$ " from 2 to $n-1$ 


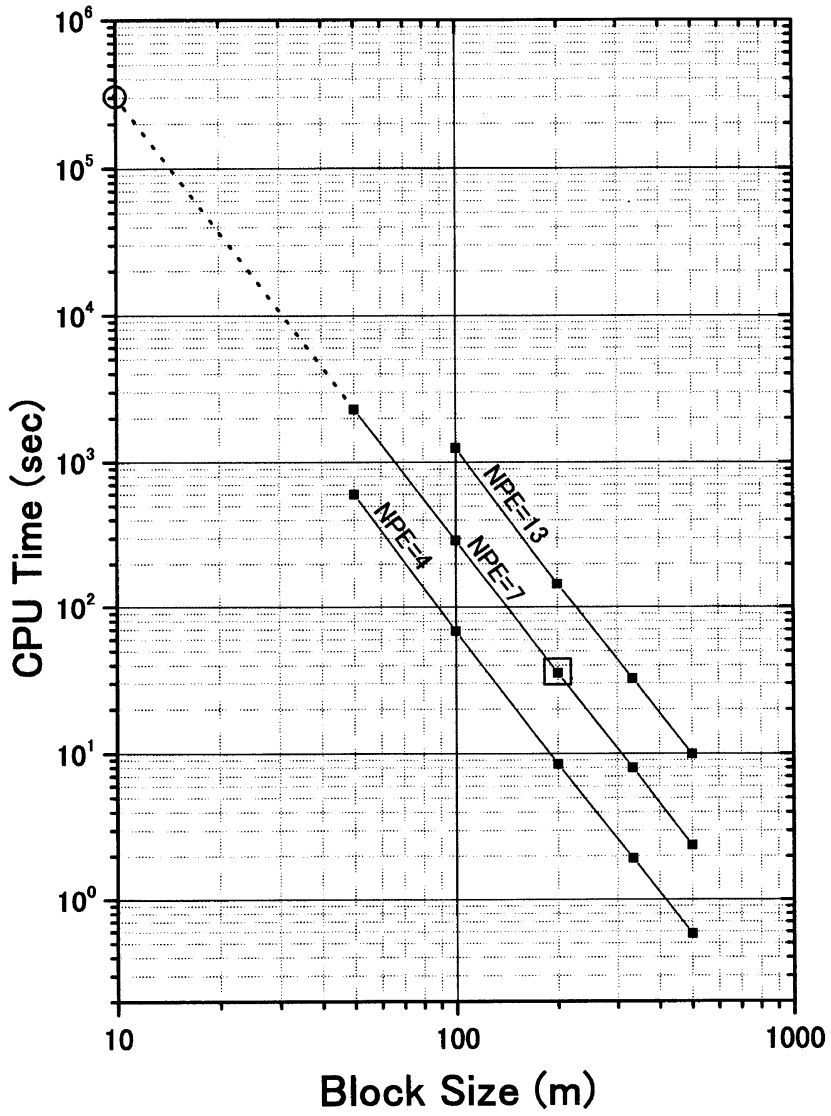

Fig. 6. CPU time as a function of the number of nodes per edge (NPE) and block size. The open circle and open square respectively indicate the CPU time for block sizes of $10 \mathrm{~m}$ and $200 \mathrm{~m}$ with NPE $=7$.

2-5: Calculate the "new" travel time from $N_{1}$ to $N_{n}$ using the results of 2-4

2-6: Compare the "new" travel time with the "old" travel time and if the "new" one is larger then terminate the procedure and take the old one as the final travel time; otherwise exchange the value of "new" and "old" and repeat the procedure from $2-4$

The appropriate block size and node configuration in Step 1 are determined from a few trials, taking into account the heterogeneous size of the velocity structure and the accuracy required.

When an abrupt change in the velocity field is anticipated the search range for $N_{i}$ in Step 2-4 should be kept small (e.g., the block size) because the travel time between the nodes is calculated using the trapezoidal rule.

Step 2 connects the stations and the seismic sources to the nodes for the shortest path calculation, and smoothes the zigzag ray path of Step 1. With real data this function of Step 2 is very important because, in general, the coordinates of stations and seismic sources do not coincide with the nodes of the shortest path calculation.

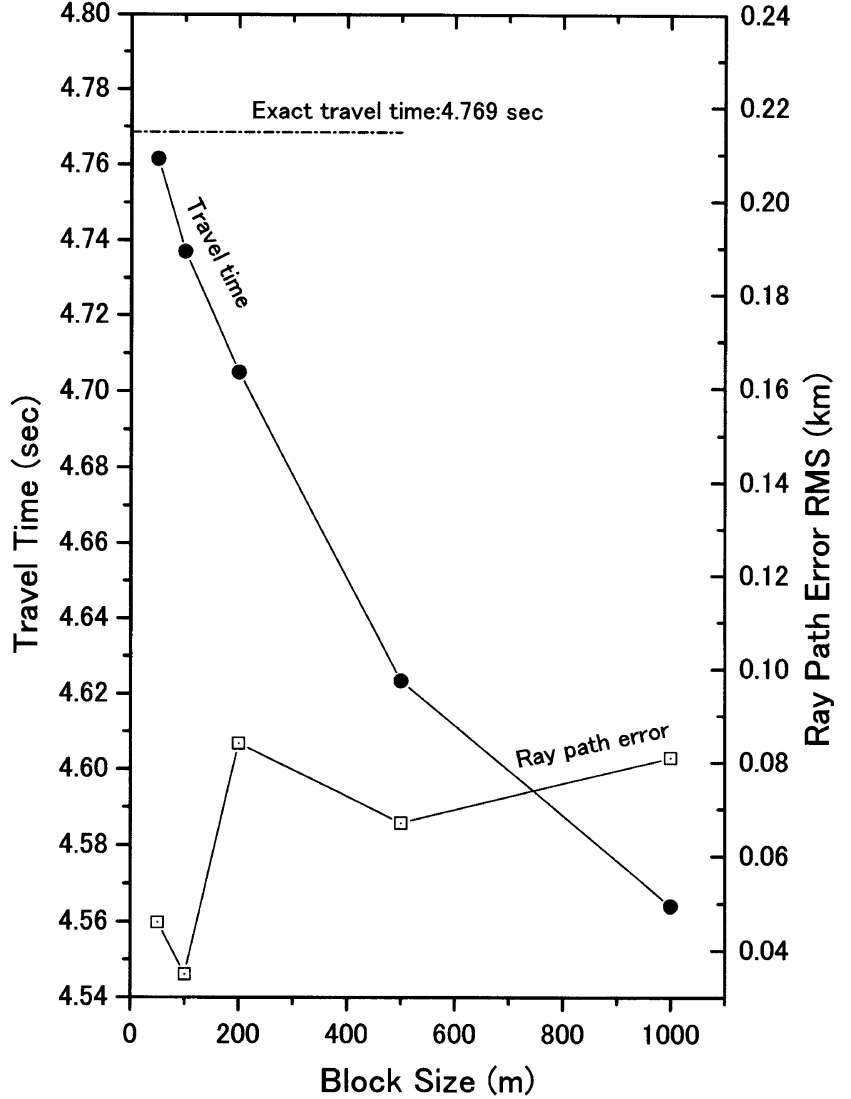

Fig. 7. The travel time and rms ray path error, by shortest path calculation with simplex method for a heterogeneous velocity model, as a function of block size with NPE $=7$.

\section{Test Results}

\section{Example 1}

Constant velocity gradient model

In this section the validity of this hybrid calculation scheme is confirmed by using the synthetic velocity structure in which the exact ray path and the travel time are obtained theoretically. Figure 2 shows the adopted velocity structure and the layout of the source and the station. The travel time and the ray path loci for this velocity structure are easy to calculate (e.g., Sheriff and Geldart, 1995). Using this velocity structure, errors in the travel time and ray path are examined, together with the efficiency of the scheme.

An example showing improvement of the ray path and the travel time using this hybrid scheme with simplex method is given in Fig. 3. In this case a large block size of $500 \mathrm{~m}$ is adopted to highlight the effect of the hybrid scheme. Figures 3(a) and (b) are respectively the results of Steps 1 and 2. The travel time with relative errors according to the shortest path ray tracing (SPR) and SPR with the simplex method are shown in Fig. 4 as a function of the number of nodes (NPE) and the block size. When the NPE number is greater than four the relative errors in the travel time are reduced significantly. For example, relative errors in the travel time for $200 \mathrm{~m}$ block size are $1.0 \sim 1.3 \%$ by the shortest path ray tracing (SPR) scheme alone, but are reduced to $0.02 \sim 0.06 \%$ by the hybrid scheme with simplex method.

The degree of improvement of the ray path loci is evaluated 
(a)

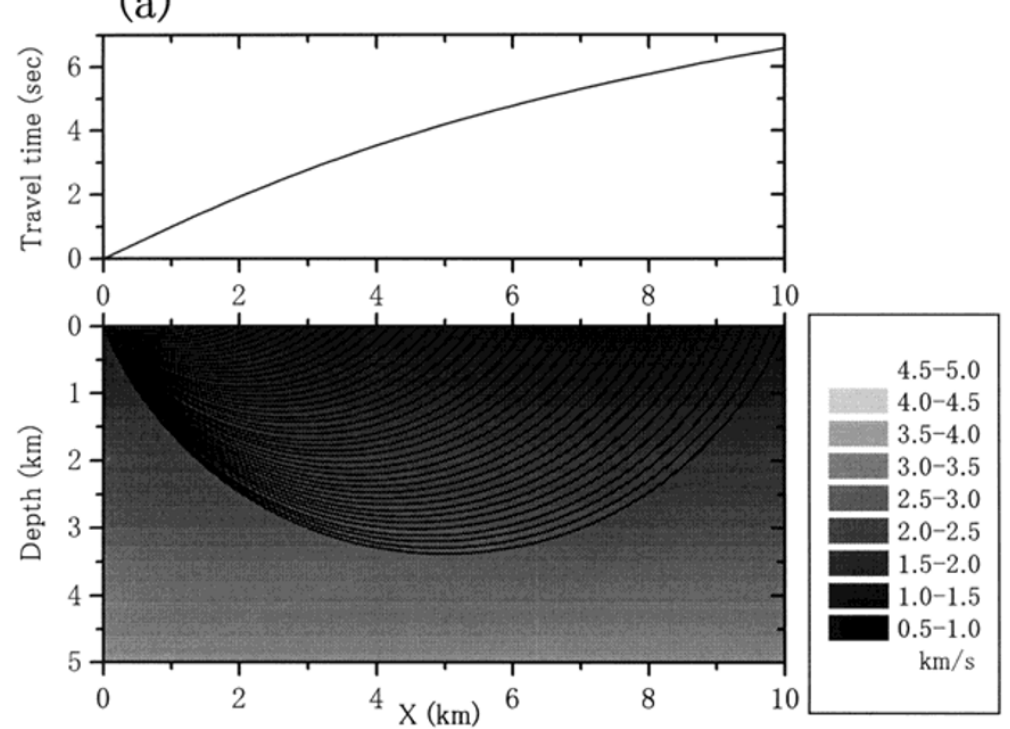

(b)

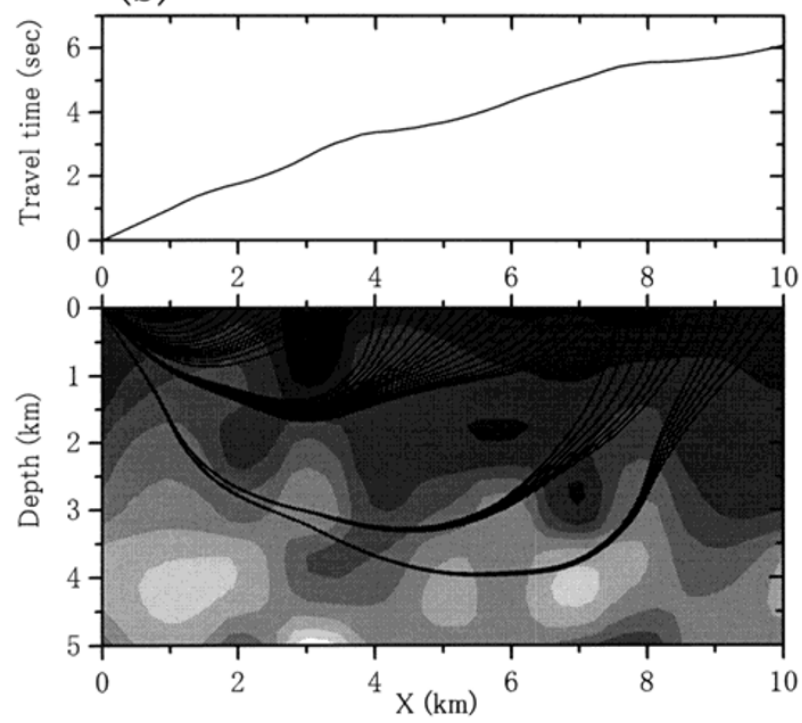

Fig. 8. Examples of travel times and ray paths for a 2-D velocity structure. (a) Travel times and ray paths for a constant velocity gradient. (b) Travel times and ray paths with lateral velocity variation.

using the ray dissimilarity defined by Fisher and Lees (1993);

$$
\text { Ray dissimilarity }=\left(\sum_{n=0}^{100} \frac{\left|p_{n}-q_{n}\right|^{2}}{101}\right)^{\frac{1}{2}}
$$

where $p_{n}$ and $q_{n}$ are points along the two rays whose travel times are equal to $n / 100$ of the full rays' travel times. The ray dissimilarity with respect to the exact ray path loci and the root mean square (rms) ray path errors are shown in Fig. 5. Significant improvement in the ray path loci is achieved by the present hybrid scheme with the simplex method.

The efficiency of the hybrid scheme with the simplex method is now examined. The accuracy required for travel times in tomography is between $1 \%$ (Fischer and Lees, 1993) and $0.1 \%$ (Klimeš and Kvasnička, 1994). From the relative error in the travel time as a function of block size in Fig. 4, a block size of $10 \mathrm{~m}$ or less may be required to give a relative error of $0.1 \%$.

Figure 6 shows the CPU time taken as a function of block size and the number of nodes per edge (NPE). Calculations are performed with an ordinary personal computer with a single CPU (K6-II, $400 \mathrm{MHz}$ ), $512 \mathrm{MB}$ memory space and the Windows 98 operating system. When the NPE is 7, the CPU time for $10 \mathrm{~m}$ block size is estimated to be about $3 \times 10^{5} \mathrm{~s}$ (open circle in Fig. 6), whereas for block size $200 \mathrm{~m}$ the CPU time is $34 \mathrm{~s}$ (open square in Fig. 6). The relative error for a $200 \mathrm{~m}$ block size is reduced to less than $0.1 \%$ by the hybrid scheme with the simplex method (Fig. 4). The relative error for a $10 \mathrm{~m}$ block size by SPR alone is estimated to be $0.1 \%$ or more, as mentioned above. Since the CPU time for the optimization using the simplex method is negligible (about 1 second), it is concluded that the efficiency of the orders of about $4\left(3 \times 10^{5} / 3.5 \times 10\right)$ is achieved by the hybrid scheme with the simplex method. 


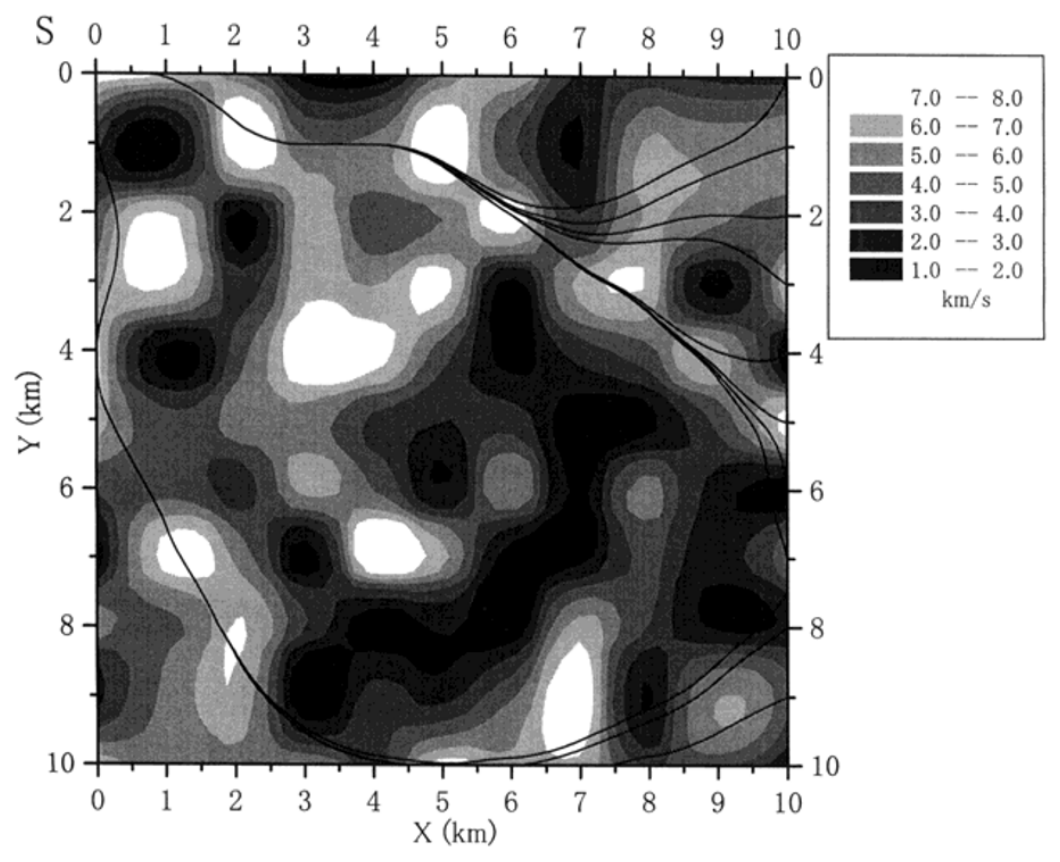

Fig. 9. Ray paths for a strongly heterogeneous 2-D velocity structure.
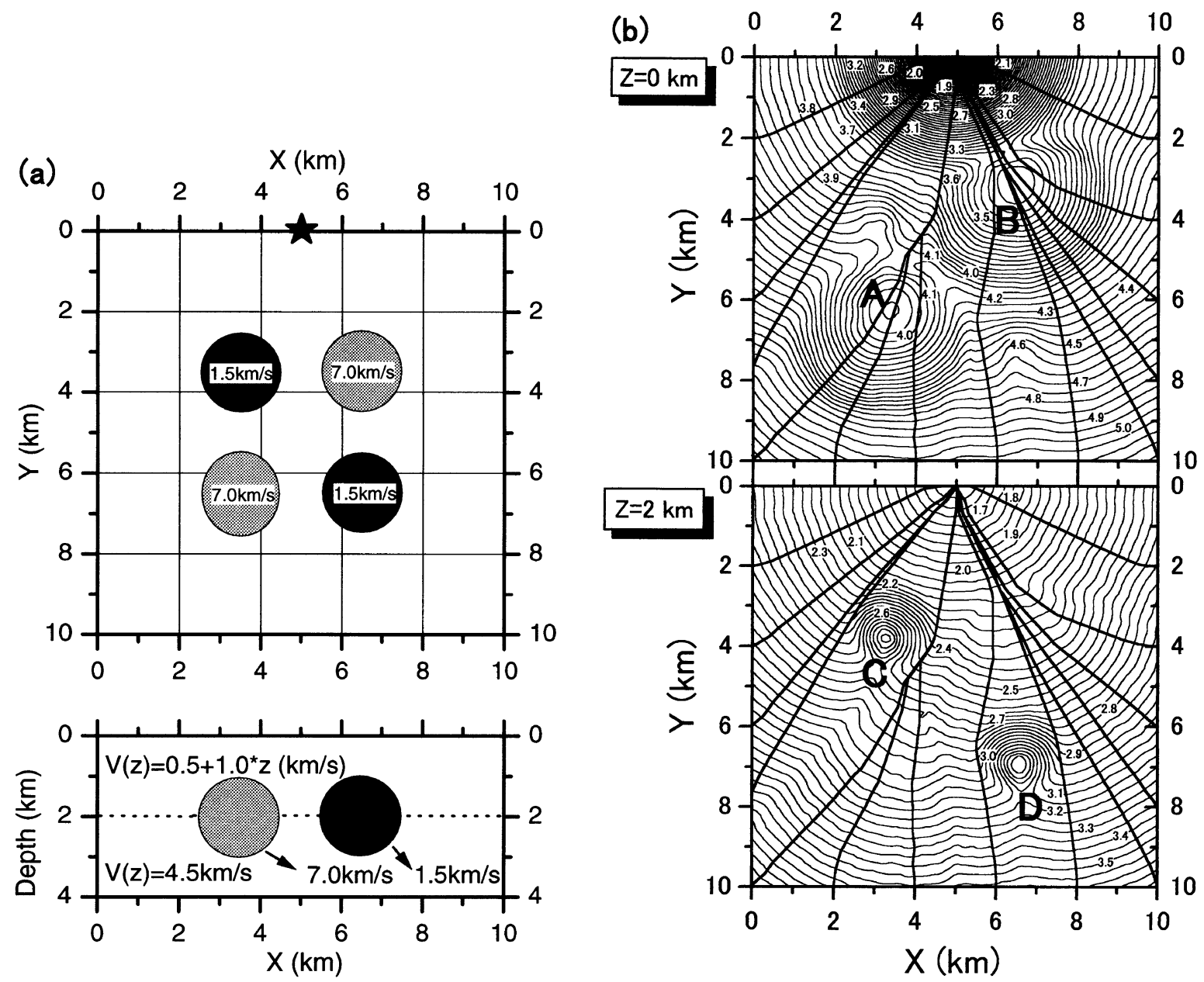

Fig. 10. Examples of the propagation of a wave front and ray paths calculated for a 3-D heterogeneous velocity structure. (a) Assumed velocity structure. Top: Horizontal projection. Solid star at $(5,0,0)$ indicates the source point. Bottom: Vertical projection. (b) Wave fronts (thin lines) and ray paths (thick lines) at the surface $(z=0 \mathrm{~km})$ (top figure) and intermediate depth $(z=2 \mathrm{~km})$ (bottom figure). The points A and B on the surface behave as secondary sources (Huygens' priciple). 

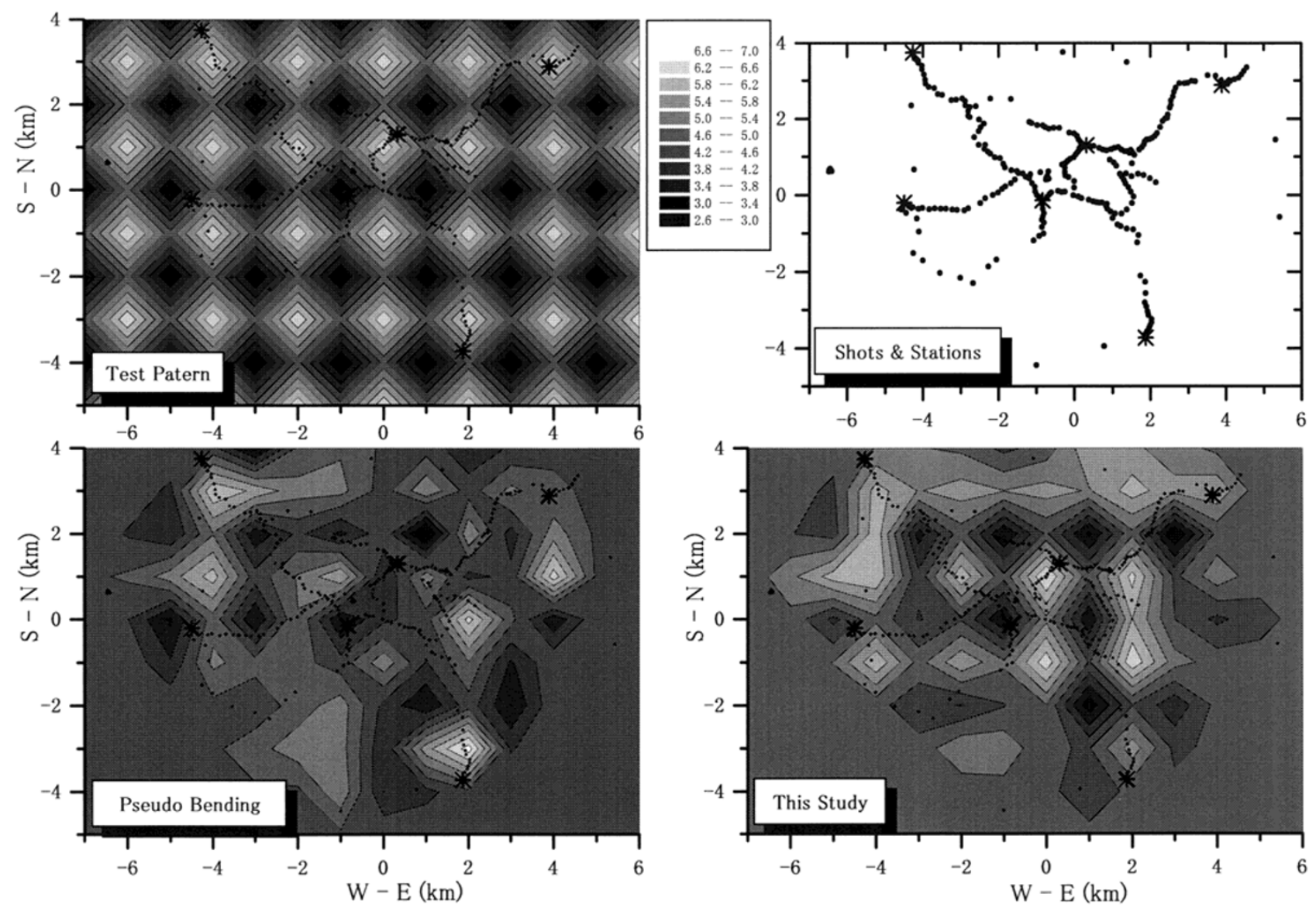

Fig. 11. Results of the checkerboard test for travel time tomography using the shots and stations configuration (top right) of Project ASO98 (Seismic explosions) (Sudo, 1999). Solid dots and asterisks indicate the stations and shots respectively. Checkerboard pattern (top left) is for a depth of $1 \mathrm{~km}$. A bottom left and bottom right are the results of velocity inversion with a pseudo bending ray tracer and the present ray tracer, respectively. Superior velocity recover is achieved by inversion with the present ray tracer.

\section{Example 2}

\section{Discontinuous velocity model}

The travel time between the nodes is calculated using the trapezoidal rule. This causes some travel time errors in a discontinuous velocity field. However, the error is reduced by using a small block size. As an example, the velocity model $V(z)=1.0+0.5 * z \mathrm{~km} / \mathrm{s}(0 \leq z<2 \mathrm{~km})$ and $V(z)=3 \mathrm{~km} / \mathrm{s}(2 \leq z)$ was tested, where $z$ is the depth in $\mathrm{km}$ and the source and station configurations are the same as in Fig. 2. The travel time and ray dissimilarity by the shortest path calculation with the simplex method and on NPE of 7 are shown in Fig. 7 as a function of block size. A travel time error less than $0.1 \%$ and ray path error less than $0.05 \mathrm{~km}$ is obtained with the block size of $50 \mathrm{~m}$. In the constant gradient velocity model (example 1), this accuracy is achieved by the larger block size of $200 \mathrm{~m}$. The CPU time for a block size of $50 \mathrm{~m}$ is about two orders of magnitude larger than for a block size of $200 \mathrm{~m}$ (Fig. 6).

\section{Example 3}

Heterogeneous velocity model in 2-D

Examples of ray paths in a 2-D heterogeneous velocity structure are shown in Figs. 8(b) and 9. Ray paths for the constant velocity gradient are shown for comparison in Fig. 8(a). The velocity of Fig. $8(\mathrm{a})$ is given by $V(z)=1.0+0.5 * z$, where $z$ is the depth in $\mathrm{km}$. The velocity structure in Fig. 8(b) is obtained from the velocity structure of Fig. 8(a) by imposing a random velocity fluctuation of maximum amplitude $40 \%$ at all grids of $1 \mathrm{~km}$ spacing. The velocity structure of Fig. 9 is obtained by imposing random values between 1 and $8 \mathrm{~km} / \mathrm{s}$ on grids of $1 \mathrm{~km}$ spacing. It can clearly be seen that the seismic rays pass preferentially through high velocity areas and avoid low velocity areas.

\section{Example 4}

Heterogeneous velocity model in 3-D

Examples of wave fronts and ray paths for a heterogeneous 3-D velocity structure (Fig. 10(a)) are shown in Fig. 10(b). Seismic waves passing through a high velocity zone arrive at points A and B in Fig. 10(b). The points A and B then behave as new wave sources; this phenomenon is known as Huygens' principle.

\section{Discussion}

The performance of the present ray tracer for travel time tomography was also examined by means of the checkerboard test. The configuration of the shots and the stations of Project ASO 98 (Seismic explosions) (Sudo, 1999) were used. The velocity structure was made by adding $\pm 40 \%$ fluctuation to the regional velocity structure obtained by Sudo (1991).

The results of the checkerboard test are shown in Figs. 11 


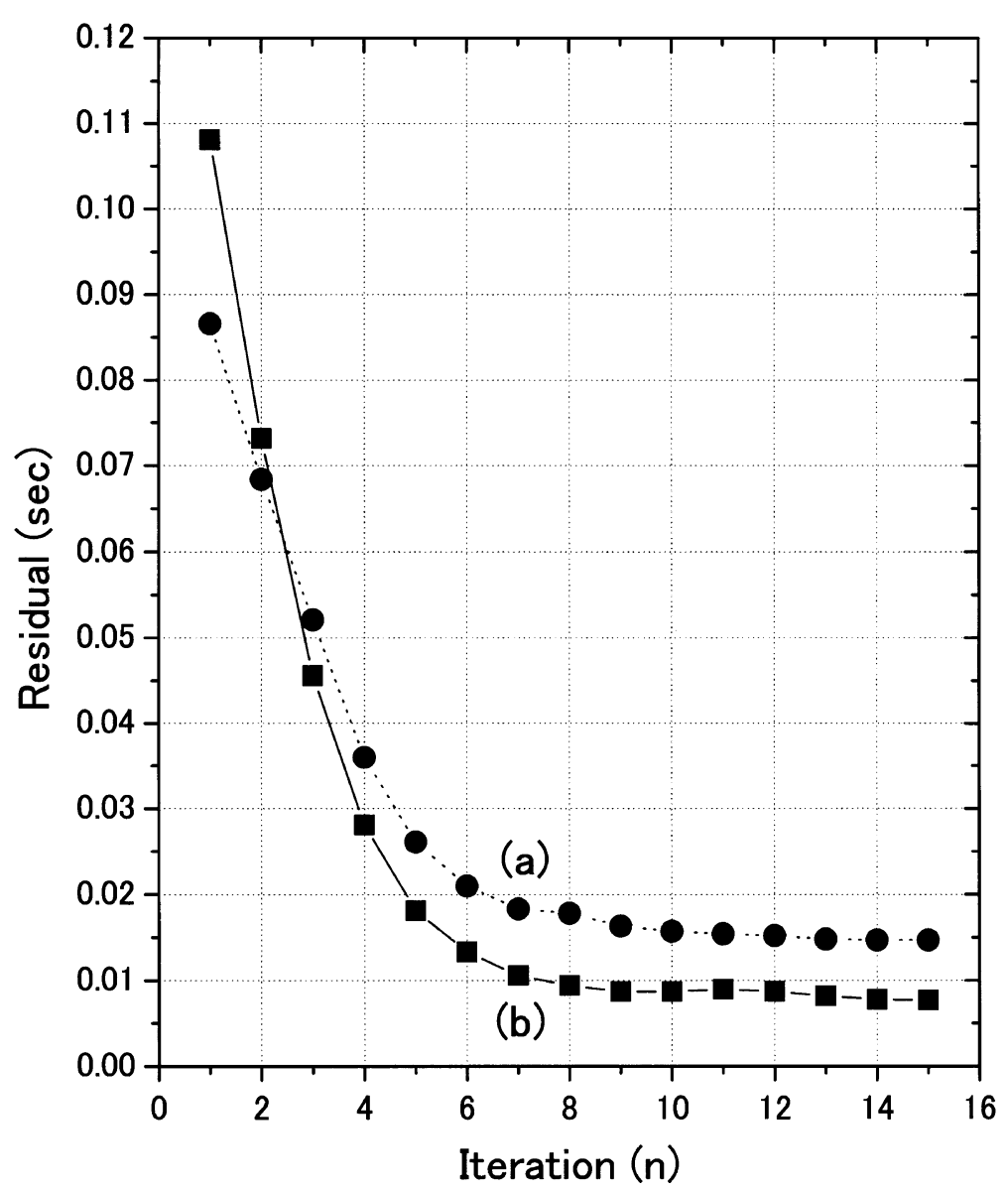

Fig. 12. Travel time residuals of the checkerboard test shown in Fig. 11. Comparison of inversion with pseudo bending (a) and inversion with the present ray tracer (b) clearly shows that travel time residuals are halved by the present ray tracer.

and 12. Results of the inversion with pseudo bending ray tracing (Um and Thurber, 1987) are also shown for comparison. Velocity inversion with the present (SPR with simplex method) shows much better velocity retrieval. The present ray tracer is consequently recommended for the velocity inversion in the heterogeneous velocity structure.

\section{Conclusions}

Of existing ray tracers, the shortest path calculation method is the most robust for strong velocity heterogeneities. Its efficiency, however, is not sufficient for practical applications such as travel time tomography. To improve the accuracy and efficiency of the calculation, the node configuration for a 3-D medium and the hybrid calculation scheme are proposed. The hybrid calculation scheme employing the shortest path calculation with the simplex method, both improves the efficiency of the calculation and resolves the inconsistency between the coordinates of stations or seismic sources and the nodes for the shortest path calculation.

Tests of the proposed method by synthetic data and a checkerboard test for velocity inversion show the effectiveness of this ray tracer in highly heterogeneous velocity structures.

Acknowledgments. I thank Professor K. Ishihara (Director of Sakurajima Volcano Research Center, DPRI, Kyoto University) for reading the manuscript and for constructive suggestions. My sin- cere thanks are due to Associate Professor I. Nakanishi (Department of Geophysics, Graduate School of Science, Kyoto University) for valuable comments and suggestions. I also thank to my colleagues in the Sakurajima Volcano Research Center DPRI, Kyoto University for their help and discussions. A suggestion by Dr. Sakai at ERI, University of Tokyo, regarding the discontinuous velocity model is appreciated. Valuable suggestions were also provided by the editor, Professor T. Tanimoto, Geological Sciences, University of California at Santa Barbara. Preliminary calculations to develop the calculation scheme were carried out using a high performance computer provided by the Unzen Volcano Scientific Drilling Project.

\section{References}

Cheng, N. and L. House, Minimum traveltime calculation in 3-D graph theory, Geophysics, 61, 1895-1898, 1996.

Dijkstra, E. W., A note on two problems in connection with graph, Numer. Math., 1, 269-271, 1959.

Fisher, R. and J. M. Lees, Shortest path ray tracing with sparse graphs, Geophysics, 58, 987-996, 1993.

Gallo, G. and S. Pallottino, Shortest path methods: A unifying approach: Mathematical Programming Study, 26, 38-64, 1986.

Klimeš, L. and M. Kvasnička, 3-D network ray tracing, Geophys. J. Int., 116, 726-738, 1994.

Moser, T. J., Shortest path calculation of seismic rays, Geophysics, 56, 59$67,1991$.

Nakanishi, I. and K. Yamaguchi, A numerical experiment on nonlinear image reconstruction from the first-arrival times for two-dimensional island arc structure, J. Phys. Earth, 34, 195-201, 1986.

Nelder, J. A. and R. Mead, A simplex method for function minimization, Computer Journal, 7, 308-313, 1965.

Podvin, P. and I. Lecomte, Finite difference computation of traveltimes in very contrasted velocity models: a massively parallel approach and its 
associated tools, Geophys. J. Int., 105, 271-284, 1991.

Press, W. H., S. A. Teukolsky, W. T. Vetterling, and B. P. Flannery, Numerical Recipes in Fortran: the art of scientific computing, 2nd edition, pp. 402 406, Cambridge University Press, 1992.

Reshef, M. and D. Kosloff, Migration of commom-shot gathers, Geophysics, 51, 324-331, 1986.

Saito, H., Travel times and ray paths of first arrival seismic waves: computation method based on Huygens' principle, in Expanded abstracts, 59th Annual Int, SEG Meeting, pp. 244-247, Soc. Explor. geophys., Tulsa, Oklahoma, 1989.

Saito, H., 3-D ray tracing method based on Huygens' principle, in Expanded abstracts, 60th Annual Int, SEG Meeting, pp. 1024-1027, Soc. Explor. geophys., Tulsa, Oklahoma, 1990.

Sheriff, R. E. and L. P. Geldart, Exploration Seismology Second Edition, pp. 93-95, Cambridge University Press, 1995.

Sudo, Y., An attenuating structure beneath the Aso Caldera determined from the propagation of seismic waves, Bull. Volcanol., 53, 99-111, 1991. Sudo, Y., Project Aso98 (Seismic explosions): Seismic explosion around active crater, Abstracts 1999 Japan Earth and Planetary Science Joint Meeting, Vb-022, 1999.

Um, J. and C. Thurber, A fast algorithm for two point seismic ray tracing, Bull. Seism. Soc. Am., 77, 972-986, 1987.

van Trier, J. and W. W. Symes, Upwind finite-difference calculation of traveltimes, Geophysics, 56, 812-821, 1991.

Vidale, J., Finite-difference calculation of traveltimes, Bull. Seis. Soc. Am. 78, 2062-2076, 1988.

Zhang, J. and M. N. Toksöz, Nonlinear refraction travel time tomography, Geophysics, 63, 1726-1737, 1998.

K. Nishi (e-mail: west@svo.dpri.kyoto-u.ac.jp) 